\title{
Behavior Choice of Game Parties under the Interference of Cognition in the Game between Coal Miners and Supervisors
}

\author{
Jun Liu ${ }^{1},{ }^{1}$ Chen Wang, ${ }^{2}$ Xinhua Wang, ${ }^{1}$ and Qingli $\mathrm{Li}^{3}$ \\ ${ }^{1}$ College of Economics and Management, Shandong University of Science and Technology, Shandong, Qingdao 266590, China \\ ${ }^{2}$ IIEM\&CCII, Beijing 100029, China \\ ${ }^{3}$ School of Economics and Management, Taishan University, Shandong Tai'an 271021, China
}

Correspondence should be addressed to Jun Liu; flybird45@tsu.edu.cn

Received 23 January 2021; Revised 14 March 2021; Accepted 2 April 2021; Published 14 April 2021

Academic Editor: Harish Garg

Copyright (c) 2021 Jun Liu et al. This is an open access article distributed under the Creative Commons Attribution License, which permits unrestricted use, distribution, and reproduction in any medium, provided the original work is properly cited.

\begin{abstract}
In the game between coal miners and supervisors, the behavior choices of the game parties will be affected by cognitive factors. The analysis of the behavior choices of coal miners and supervisors under the influence of cognitive factors is helpful for the design of violation behavior control strategies. Firstly, a description method of subjective cognition based on the mathematical method of quantum theory is designed. Secondly, taking the subjective cognition of coal miners and supervisors as a random variable, a behavior evolution model with random variables is constructed. Thirdly, the impact of subjective cognition on the behavior choices of coal miners and supervisors is analyzed. Finally, the violation behavior control strategy is designed. It is found that when the violation probability decreases to a certain extent, the probability of supervision will change from the increase to decrease. When the probability of supervision decreases to a certain extent, the violation probability will change from the decrease to increase. Fluctuations in cognitive state can affect the change process of violation probability and supervision probability. The behavior control strategy designed according to the behavior evolution model can control the violation behavior in the situation of cognitive state fluctuation.
\end{abstract}

\section{Introduction}

The violation behavior of coal miners is an important factor affecting safety production [1-4]. Cognition is a psychological factor affecting coal miners' behavior $[5,6]$. Cognition can be affected by some factors, such as mental pressure [7-9]. The relationship between pressure and cognitive competence is an inverted U-shaped curve [7]. In the game between coal miners and supervisors, the game parties' cognition usually has bias. For example, coal miners may underestimate the risk of violation behavior and overestimate its utility. In China, the education level of coal miners is generally low, and their cognitive ability is limited. Under the influence of production pressure, life pressure, and other stochastic factors, their cognition of the utility of violation behavior tends to fluctuate. To analyze the impact of cognition on the game parties' behavior choice in the game between coal miners and supervisors, we will design a description method of subjective cognition based on quantum theory and build a behavior evolution model considering the influence of subjective cognition based on evolutionary game theory.

According to the behavioral decision theory, decisionmakers are not entirely rational. Most people are also not completely selfish $[10,11]$. The human brain can conduct complicated calculations and and have a complicated memory. However, biological and social evolutions equip us with a set of mental tools. We always use this set of tools to make decisions [12]. As a mental factor, cognition is in close relation to decision-making [13]. To better describe the impact of cognition on decision-making, some authors have introduced quantum theory to the decision-making field and made many achievements [14-24]. Research on cognitive psychology indicates that quantum probability can describe the decision-making of human beings in an elegant framework [14]. This provides another method for 
computing probabilities without falling into the restrictions that classical probability has regarded to model cognitive systems and decision-making [15-18]. The quantum-like decision-making model proposed by Moreira and Wichert can recognize the optimal decision in their belief space [19]. Bohr thinks that the mental process of people is similar to the course of quantum physics in many aspects and that quantum theory can be used as a mathematical tool for solving problems involving the thinking process of human beings [20]. The mathematical methods in quantum theory can explain humans' cognitive processes, including judgment, decision-making, conceptmaking, inference, memory, and perception [21]. Busemeyer and Trueblood put forward six reasons for applying quantum theory to human cognition [22]. Vaio thinks that the mathematical methods in quantum theory can explain the behavior of violating logic and help in the establishment of mental and cognitive models [23]. The above study shows that it is feasible to describe the cognitive state of the game parties by making use of the mathematical methods in quantum theory.

In terms of the game problem between the stakeholders in the production system of a coal mine, Lu and Wang conducted relevant research on the fractional supervision game model [24], the multiple evolution game model [25], the stability of the evolution game system [26], and the evolution dynamics model based on the delay and impulsive differential equation [27]. Furthermore, some authors have analyzed the dynamic process of a multiplayer game in a coal mine safety supervision system [28-30]. Some authors have also analyzed the symmetry of game benefits between managers and coal miners [31]. Moreover, they have also analyzed the game relationships between the stakeholders in the coal mine safety production system $[32,33]$. These researches all provide a reference for the analysis of the behavior of game parties.

The traditional game theory is built on the assumption that the game parties are completely rational [34]. It does not consider the irrational decision-making behaviors caused by some mental factors such as cognition. Compared with traditional game theory, the evolution game theory deems that the game parties will learn in the game and seek a relatively good strategy by trial and error instead of modelling people into superrational game parties [35-37]. The evolution game theory can be used to analyze the evolution process of the decision-making behavior of game parties [38-40]. For example, based on the evolution game theory, Shia et al. analyzed the dynamic evolutionary process of evacuation decisionmaking for different decision-makers in a wildfire event [41], and $\mathrm{Du}$ et al. analyzed the evolution process of stakeholders' decision-making behaviors in construction and demolition waste management [42]. We will use evolutionary game theory to establish the behavior evolution model and then use the behavior evolution model to analyze the behavior evolution process of game parties in the game between coal miners and supervisors under the fluctuation of cognitive state and finally design a behavior control strategy.

\section{Behavior Evolution Model considering the Interference of Cognition}

2.1. Model Assumption. The relevant assumptions in the game between coal miners and supervisors are as follows.

Coal miners have two behaviors for selection, operating in violation of the rules and operating according to the rules, which are labelled as $A_{1}$ and $A_{2}$, respectively. The probability of choosing $A_{1}$ is $x_{1}$ and the probability of choosing $A_{2}$ is $x_{2}$, where $x_{1}+x_{2}=1$. Supervisors have two behaviors for selection, supervising and not supervising, which can be labelled as $B_{1}$ and $B_{2}$, respectively. The probability of choosing $B_{1}$ is $y_{1}$ and the probability of choosing $B_{2}$ is $y_{2}$, where $y_{1}+y_{2}=1$.

Coal miners have an incomplete cognition of $y_{1}$ and $y_{2}$. Supervisors have an incomplete cognition of $x_{1}$ and $x_{2}$. Coal miners and supervisors can make behavior choices according to their subjective judgment.

Operations in violation of rules can save time. Coal miners can obtain $u_{0}$ if they operate according to the rules. Meanwhile, if they operate in violation of rules, they can obtain $u_{0}+I$, where $I$ is the utility of saving time. If supervisors find that coal miners operate in violation of the rules, coal miners will suffer a corresponding punishment $f$. If supervisors find that coal miners operate according to the rules, coal miners will obtain the award $b$. The enterprise loss that the operation in violation of the rules leads to will be $l$. If supervisors supervise, this loss can be avoided. The supervisory cost is $c_{0}$. The game payment matrix can be shown as in Table 1.

2.2. Description of Cognitive State. In quantum theory, the quantum state can be used to represent the state of the quantum system $[43,44]$. Labelled as $|\psi\rangle$, the quantum state is the vector representation of $H_{n}$, which is the $n$-dimensional Hilbert Space. $|\cdot\rangle$ is the symbol of Dirac [43]. It can be assumed that there is a quantum state $\left.|\psi\rangle \in H_{n},|| \psi\right\rangle \mid=1$, and the general definition is shown as follows:

$$
|\psi\rangle=\sum_{j=1}^{n} c_{j}|j\rangle
$$

where $|j\rangle$ is an $n$-dimensional column vector. The $j$ th line is 1 and the other lines are $0 .|j\rangle$ is the basic state of the system. As a plural, $c_{j}$ is called the probability amplitude of $|j\rangle$. $\left|c_{j}\right|^{2}=p_{j}, c_{j}=\sqrt{p_{j}} e^{i \theta_{j}}, p_{j}$ is the probability that the basic state $|j\rangle$ appears, $e^{i \theta_{j}}=\cos \theta_{j}+i \sin \theta_{j}$, and $\theta_{j}$ is the argument. When $n=2, \quad|1\rangle=\left(\begin{array}{l}1 \\ 0\end{array}\right), \quad|2\rangle=\left(\begin{array}{l}0 \\ 1\end{array}\right)$, $c_{1}=\sqrt{p_{1}} e^{i \theta_{1}}=\sqrt{p_{1}} \cos \theta_{1}+i \sqrt{p_{1}} \sin \theta_{1}$, $c_{2}=\sqrt{p_{2}} e^{i \theta_{2}}=\sqrt{p_{2}} \cos \theta_{2}+i \sqrt{p_{2}} \sin \theta_{2}$, and $|\psi\rangle=\left(\begin{array}{l}c_{1} \\ c_{2}\end{array}\right)$. The relationship between $|1\rangle,|2\rangle$, and $|\psi\rangle$ is shown in Figure 1.

In quantum theory, $|\cdot\rangle$ and $\langle\cdot|$ are called bra and ket, respectively [43]. $\langle\cdot|$ is the conjugate transpose of $|\cdot\rangle$. For example, when $n=2,\langle\psi|=\left(c_{1}^{*} c_{2}^{*}\right)$, where $c_{1}^{*}=\sqrt{p_{1}} \cos \theta_{1}-$ 
TABle 1: Game payment matrix between coal miners and supervisors.

\begin{tabular}{ccc}
\hline & $B_{1}$ & $B_{2}$ \\
\hline$A_{1}$ & $\left(u_{A 11}=u_{0}+I-f, u_{B 11}=-c_{0}\right)$ & $\left(u_{A 12}=u_{0}+I, u_{B 21}=-l\right)$ \\
$A_{2}$ & $\left(u_{A 21}=u_{0}+b, u_{B 12}=-c_{0}\right)$ & $\left(u_{A 22}=u_{0}, u_{B 22}=0\right)$ \\
\hline
\end{tabular}

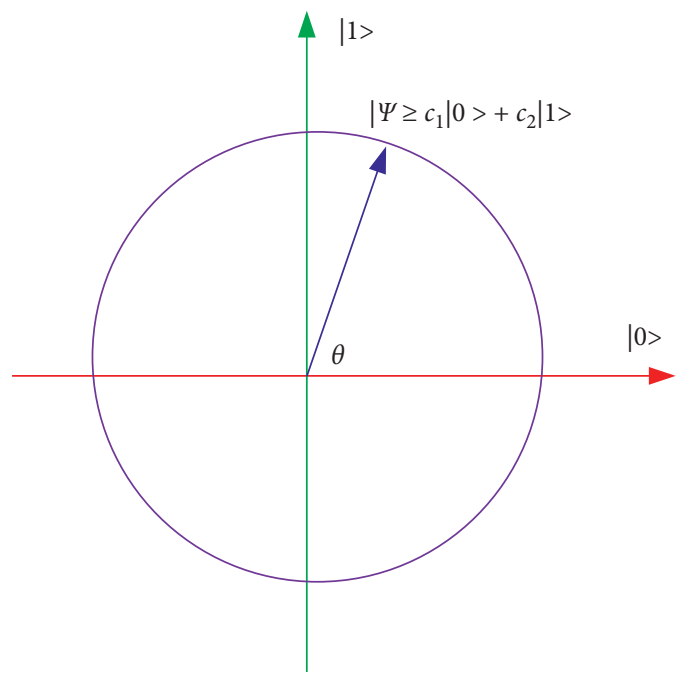

Figure 1: The relationship between $|1\rangle,|2\rangle$, and $|\psi\rangle$.

$i \sqrt{p_{1}} \sin \theta_{1}$ and $c_{2}^{*}=\sqrt{p_{2}} \cos \theta_{2}-i \sqrt{p_{2}} \sin \theta_{2} \cdot c_{1}^{*}$ and $c_{2}^{*}$ are the complex conjugates of $c_{1}$ and $c_{2} \cdot c_{1} c_{1}^{*}=p_{1}$ and $c_{2} c_{2}^{*}=p_{2}$.

$|\psi\rangle\langle\psi|$ is called a density operator. $|\psi\rangle$ $\left\langle\psi\left|=\left(\begin{array}{l}c_{1} \\ c_{2}\end{array}\right)\left(c_{1}^{*} c_{2}^{*}\right)=\left(\begin{array}{l}c_{1} c_{1}^{*} c_{1} c_{2}^{*} \\ c_{2} c_{1}^{*} c_{2} c_{2}^{*}\end{array}\right)=\left(\begin{array}{l}p_{1} c_{1} c_{2}^{*} \\ c_{2} c_{1}^{*} p_{2}\end{array}\right) . \quad\right| 1\right\rangle\langle 1|=$ $\left(\begin{array}{l}1 \\ 0\end{array}\right)\left(\begin{array}{ll}1 & 0\end{array}\right)=\left(\begin{array}{ll}1 & 0 \\ 0 & 0\end{array}\right), \quad|1\rangle\langle 2|=\left(\begin{array}{l}1 \\ 0\end{array}\right)\left(\begin{array}{ll}0 & 1\end{array}\right)=\left(\begin{array}{ll}0 & 1 \\ 0 & 0\end{array}\right)$, $|2\rangle\langle 1|=\left(\begin{array}{l}0 \\ 1\end{array}\right)\left(\begin{array}{ll}1 & 0\end{array}\right)=\left(\begin{array}{ll}0 & 0 \\ 1 & 0\end{array}\right), \quad$ and $\quad|2\rangle\langle 2|=\left(\begin{array}{l}0 \\ 1\end{array}\right)$ $\left(\begin{array}{ll}0 & 1\end{array}\right)=\left(\begin{array}{ll}0 & 0 \\ 0 & 1\end{array}\right)$. If $D=\left(\begin{array}{ll}u_{1} & u_{2} \\ u_{3} & u_{4}\end{array}\right), \operatorname{tr}(D)=u_{1}+u_{4}$ is called the trace of the matrix $D$.

According to the Dirac-Von Neumann axiom set [44], the observable quantity $D$ of the quantum system is a selfadjoint operator of $H_{n}$. That is to say that $D^{*}=D$. $D^{*}$ is the conjugate transpose of $D$. In the quantum state $|\psi\rangle$, the average value of $D$ is $D=\langle\psi|D| \psi\rangle=\operatorname{tr}(D|\psi\rangle\langle\psi|)=\operatorname{tr}(D \rho)$. $\rho=|\psi\rangle\langle\psi|$ is the density operator. $\operatorname{tr}(D \rho)$ is the trace of the matrix $D \rho$.

According to the type of expression of quantum state, Asano et al. described the cognitive process of decisionmakers and Basieva et al. described the belief state of decision-makers $[45,46]$. According to these studies, a coal miners' cognitive state about whether supervisors supervise at $t$ is shown as follows:

$$
|A(t)\rangle_{m}=\sqrt{y_{1}} e^{i \theta_{m 1}(t)}\left|1_{m}\right\rangle+\sqrt{y_{2}} e^{i \theta_{m 2}(t)}\left|2_{m}\right\rangle,
$$

where $|1\rangle_{m}=\left(\begin{array}{l}1 \\ 0\end{array}\right)$ and $|2\rangle_{m}=\left(\begin{array}{l}0 \\ 1\end{array}\right)$ represent states where the supervisors supervise and do not supervise, respectively. $\left|\sqrt{y_{1}} e^{i \theta_{m 1}(t)}\right|^{2}=y_{1}$ and $\left|\sqrt{y_{2}} e^{i \theta_{m 2}(t)}\right|^{2}=y_{2}$.

The supervisors' cognitive state about whether coal miners operate in violation of the rules at $t$ is shown as follows:

$$
|B(t)\rangle_{c}=\sqrt{x_{1}} e^{i \varphi_{c 1}(t)}|1\rangle_{c}+\sqrt{x_{2}} e^{i \varphi_{c 2}(t)}|2\rangle_{c},
$$

where $|1\rangle_{c}=\left(\begin{array}{l}1 \\ 0\end{array}\right)$ and $|2\rangle_{c}=\left(\begin{array}{l}0 \\ 1\end{array}\right)$ represent states where the coal miners operate in violation of the rules and operate according to the rules, respectively.

2.3. Subjective Cognition on the Utility of Behavior. We draw lessons from the quantum-like decision-making models proposed by Asano et al. and Basieva et al. $[45,46]$ and then construct the operator $U_{A_{1}}$ which can be used to compare $A_{1}$ with $A_{0} . A_{0}$ means that no action is taken. $u_{A 01}$ and $u_{A 02}$, which are the utilities of $A_{0}$ in the supervisory and nonsupervisory states, are both 0 . The definition of $U_{A_{1}}$ is shown as follows:

$$
U_{A_{1}}=\frac{1}{2}\left[D_{A_{0}} \longrightarrow A_{1}-D_{A_{1}} \longrightarrow A_{0}\right]
$$

where $\quad D_{A_{0}} \longrightarrow A_{1}=\sum_{k=1}^{2} \sum_{l=1}^{2}\left(u_{A 1 k}-u_{A 0 l}\right) \quad e^{i \varnothing_{l^{A 0}} \rightarrow k^{A 1}}|k\rangle_{m}$ $\left\langle\left. l\right|_{m} \text { and } D_{A_{1}} \longrightarrow A_{0}=\sum_{l=1}^{2} \sum_{k=1}^{2}\left(u_{A 0 l}-u_{A 1 k}\right) e^{i \varnothing_{k^{A 1}} \rightarrow l^{A 0}} \mid l\right\rangle_{m}$ $\left\langle\left. k\right|_{m} . \mid k\right\rangle_{m}\left\langle\left. l\right|_{m}\right.$ which is the operator of state transition represents that the state turns to $|k\rangle_{m}$ from $|l\rangle_{m} \cdot u_{A 1 k}-u_{A 0 l}$ represents that when $|l\rangle_{m}$ turns to $|k\rangle_{m}$, the coal miner will obtain $u_{A 1 k}$ while losing the chance to obtain $u_{A 0 l}$ in the meantime. $u_{A 1 k}-u_{A 0 l}$ is called relative utility. $D_{A_{0}} \longrightarrow A_{1}$ is the utility that $A_{1}$ is relative to $A_{0}$ and $D_{A_{1}} \longrightarrow A_{0}$ is the utility that $A_{0}$ is relative to $A_{1} \cdot \varnothing_{l^{A 0} \longrightarrow k^{A 1}}$ is the argument. $\varnothing_{l^{A 0} \longrightarrow k^{A 1}}=-\varnothing_{k^{A 1} \longrightarrow l^{A 0}}$ represents that $u_{A 1 k}-u_{A 0 l}$ and $u_{A 0 l}-u_{A 1 k}$ are a utility comparison with an opposite direction. When $l=k, \varnothing_{l^{A 0}} \longrightarrow k^{A 1}=0$ represents the utility comparison in the same basic state. When $l \neq k, \varnothing_{l^{A 0}} \longrightarrow k^{A 1}=$ $(\pi / 2)$ represents the utility comparison in different basic states.

At time $t$, the average value of $U_{A_{1}}$ is $U_{A_{1}}(t)=\operatorname{tr}\left(U_{A_{1}}\right.$ $\left.{ }^{*} \rho_{A}(t)\right)=(1 / 2) \operatorname{tr}\left(D_{A_{0}} \longrightarrow A_{1}{ }^{*} \rho_{A}(t)-D_{A_{1}} \longrightarrow A_{0}{ }^{*} \rho_{A}(t)\right)$. The density operator $\rho_{A}(t)=|A(t)\rangle_{m}\left\langle\left. A(t)\right|_{m}\right.$ is shown as follows:

$\rho_{A}(t)=\left(\begin{array}{cc}\sqrt{y_{1} y_{1}} e^{i\left(\theta_{m 1}(t)-\theta_{m 1}(t)\right)} & \sqrt{y_{1} y_{2}} e^{i\left(\theta_{m 1}(t)-\theta_{m 2}(t)\right)} \\ \sqrt{y_{2} y_{1}} e^{i\left(\theta_{m 2}(t)-\theta_{m 1}(t)\right)} & \sqrt{y_{2} y_{2}} e^{i\left(\theta_{m 2}(t)-\theta_{m 2}(t)\right)}\end{array}\right)$.

The matrix forms of $D_{A_{0}} \longrightarrow A_{1}$ and $D_{A_{1}} \longrightarrow A_{0}$ are shown as follows: 
$\begin{aligned} & D_{A_{0}} \longrightarrow A_{1}=\left(\begin{array}{ll}\left(u_{A 11}-u_{A 01}\right) e^{i \varnothing_{1} A 0 \rightarrow 1^{A 1}} & \left(u_{A 11}-u_{A 02}\right) e^{i \varnothing_{2} A 0 \rightarrow 1^{A 1}} \\ \left(u_{A 12}-u_{A 01}\right) e^{i \varnothing_{1} A 0 \rightarrow 2^{A 1}} & \left(u_{A 12}-u_{A 02}\right) e^{i \varnothing_{2^{A 0} \rightarrow 2^{A 1}}}\end{array}\right), \\ & D_{A_{1} \longrightarrow A_{0}}=\left(\begin{array}{ll}\left(u_{A 01}-u_{A 11}\right) e^{i \varnothing_{1} A 1 \rightarrow 1^{A 0}} & \left(u_{A 01}-u_{A 12}\right) e^{i \varnothing_{2^{A 1}} \rightarrow 1^{A 0}} \\ \left(u_{A 02}-u_{A 11}\right) e^{i \varnothing_{1} A 1 \rightarrow 2^{A 0}} & \left(u_{A 02}-u_{A 12}\right) e^{i \varnothing_{2^{A 1}} \rightarrow 2^{A 0}}\end{array}\right),\end{aligned}$

and $U_{A_{1}}(t)$ is shown as follows:

$U_{A_{1}}(t)=\sum_{k=1}^{2} \sum_{l=1}^{2}\left[\left(u_{A 1 k}-u_{A 0 l}\right)^{*} \sqrt{y_{k} y_{l}} \cos \left(\theta_{l^{A 0}} \longrightarrow k^{A 1}(t)\right)\right]$,

where $\theta_{l^{A 0} \longrightarrow k^{A 1}}(t)=\varnothing_{l^{A 0} \longrightarrow k^{A 1}}+\theta_{m k}(t)-\theta_{m l}(t) ; u_{A 0 l}=0$. $U_{A_{1}}(t)$ can be also expressed as

$$
U_{A_{1}}(t)=\sum_{k=1}^{2} p_{k}^{\prime}(t) u_{A 1 k}
$$

where $p_{k}^{\prime}(t)=\left[\sum_{l=1}^{2} \sqrt{y_{l}} \cos \left(\theta_{l^{A 0}} \longrightarrow k^{A 1}(t)\right)\right] \sqrt{y_{k}} \cdot p_{1}^{\prime}(t)$ and $p_{2}^{\prime}(t)$ are shown as follows:

$$
p_{1}^{\prime}(t)=y_{1} \cos \left(\theta_{1^{A 0} \longrightarrow 1^{A 1}}(t)\right)+\sqrt{y_{1} y_{2}} \cos \left(\theta_{2^{A 0} \longrightarrow 1^{A 1}}(t)\right),
$$

$$
p_{2}^{\prime}(t)=y_{2} \cos \left(\theta_{2^{A 0} \longrightarrow 2^{A 1}}(t)\right)+\sqrt{y_{2} y_{1}} \cos \left(\theta_{1^{A 0} \longrightarrow 2^{A 1}}(t)\right),
$$

where $p_{1}^{\prime}(t)$ and $p_{2}^{\prime}(t)$ can be seen as the subjective judgment of coal mine workers on whether supervisors try hard to supervise. $U_{A_{1}}(t)$ is the utility perception of $A_{1}$.

In $\quad(9) \quad$ and $(10), \quad \theta_{1^{A 0}} \longrightarrow 1^{A 1}(t)=\varnothing_{1^{A 0} \longrightarrow 1^{A 1}}$ $+\theta_{m 1}(t)-\theta_{m 1}(t)=0, \quad \theta_{2^{A 0} \longrightarrow 2^{A 1}}(t)=\varnothing_{2^{A 0} \longrightarrow 2^{A 1}}+\theta_{m 2}$ $(t)-\theta_{m 2}(t)=0, \quad \theta_{2^{A 0}} \longrightarrow 1^{A 1}(t)=\varnothing_{2^{A 0}} \longrightarrow \quad 1^{A 1}+\theta_{m 1}(t)-$ $\theta_{m 2}(t)=(\pi / 2)+\theta_{m 1}(t)-\theta_{m 2}(t), \quad$ and $\quad \theta_{1^{A 0}} \longrightarrow 2^{A 1}(t)=$ $\varnothing_{1^{A 0} \longrightarrow 2^{A 1}}+\theta_{m 2}(t)-\theta_{m 1} \quad(t)=(\pi / 2)+\theta_{m 2}(t)-\theta_{m 1}$ $(t) \cdot \cos \left(\theta_{1^{A 0} \longrightarrow 1^{A 1}}(t)\right) \quad=\cos \left(\theta_{2^{A 0} \longrightarrow 2^{A 1}}(t)\right)=0$ and $\cos \left(\theta_{2^{A 0} \longrightarrow 1^{A 1}}(t)\right)=-\cos \left(\theta_{1^{A 0}} \longrightarrow 2^{A 1}(t)\right)$. Equations (9) and (10) can be transformed as

$$
\begin{aligned}
& p_{1}^{\prime}(t)=y_{1}+\sqrt{y_{1} y_{2}} \cos \left(\theta_{A}(t)\right), \\
& p_{2}^{\prime}(t)=y_{2}-\sqrt{y_{2} y_{1}} \cos \left(\theta_{A}(t)\right),
\end{aligned}
$$

where $\quad \theta_{A}(t)=\theta_{2^{A 0} \longrightarrow 1^{A 1}}(t), \quad p_{1}^{\prime}(t)+p_{2}^{\prime}(t)=1, \quad$ and $\cos \left(\theta_{A}(t)\right)$ is a random variable. When $\cos \left(\theta_{A}(t)\right)=0$, $p_{1}^{\prime}(t)=y_{1}$ and $p_{2}^{\prime}(t)=y_{2}$. This represents that the coal miners' cognition of $y_{1}$ and $y_{2}$ has no bias. When $\cos \left(\theta_{A}(t)\right)>0, p_{1}^{\prime}(t)>y_{1}$, and $p_{2}^{\prime}(t)<y_{2}$, this represents that coal miners overrate $y_{1}$ and underestimate $y_{2}$. When $\cos \left(\theta_{A}(t)\right)<0, p_{1}^{\prime}(t)<y_{1}$, and $p_{2}^{\prime}(t)>y_{2}$, this represents that coal miners underestimate $y_{1}$ and overrate $y_{2}$.

According to the fluctuation in $\cos \left(\theta_{A}(t)\right)$ in a certain scope, we can describe the fluctuation of the game parties' cognitive state under the effects of different factors. Furthermore, we can introduce the parameter $\sigma$ and then use $\sigma * \cos \left(\theta_{A}(t)\right)$ to adjust the fluctuation range of the cognitive state. $\sigma$ is a real number that is larger than 0 . It can be assumed that $\cos \left(\theta_{A}(t)\right)$ is a stochastic process of the following form:

$$
\xi(t)=\cos (\omega t+U),
$$

where $\omega$ is a real number and $U$ is a random variable. For ensuring $0 \leq p_{k}^{\prime}(t) \leq 1$, the following updating is made for $p_{k}^{\prime}(t)$ :

$$
p_{k}(t)= \begin{cases}0, & p_{k}^{\prime}(t)<0, \\ 1, & p_{k}^{\prime}(t)>1, \\ p_{k}^{\prime}(t), & \text { others. }\end{cases}
$$

We let $y_{1}=0.8, y_{2}=0.2$, and $\sigma=0.1$. When $U$ obeys the uniform distribution on $[0,2 \pi], p_{1}(t)$ and $p_{2}(t)$ under the influence of $\xi(t)$ are shown in Figure 2. $p_{1}(t)$ is coal miners' subjective cognition on $y_{1}$. Under the influence of random factors, coal miners will have different judgments on $y_{1}$ at different times. Sometimes they overestimate $y_{1}$ and sometimes underestimate $y_{1}$. Overestimation and underestimation are uncertain. Processes like this are usually described by stochastic processes [47, 48]. Figure 2 is an example of using the stochastic process to describe the fluctuation of the cognitive state. As can be seen from Figure 2, $\xi(t)$ can describe the uncertain process in which coal miners sometimes overestimate $y_{1}$ and sometimes underestimate $y_{1}$.

After updating, the coal miners' subjective cognition on the utility of $A_{1}$ is

$$
U_{A_{1}}(t)=\sum_{k=1}^{2} p_{k}(t) u_{A 1 k}
$$

When $\cos \left(\theta_{A}(t)\right)>0, \quad p_{1}(t) \geq y_{1}, \quad p_{2}(t) \leq y_{2}, \quad$ and $U_{A_{1}}(t)=\left(u_{0}+I-f\right) p_{1}(t)+\left(u_{0}+I\right) p_{2}$

$(t) \leq\left(u_{0}+I-f\right) y_{1}+\left(u_{0}+I\right) y_{2}$. This represents the state where the coal miners underestimate the utility of $A_{1}$.

When $\cos \left(\theta_{A}(t)\right)<0, \quad p_{1}(t) \leq y_{1}, \quad p_{2}(t) \geq y_{2}, \quad$ and $U_{A_{1}}(t)=\left(u_{0}+I-f\right) p_{1}(t)+\left(u_{0}+I\right) p_{2}(t) \geq\left(u_{0}+\right.$

$I-f) y_{1}+\left(u_{0}+I\right) y_{2}$. This represents the state where the coal miners overrate the utility of $A_{1}$.

Asano et al. regard $\cos \left(\theta_{A}(t)\right)$ as the decision-maker's estimate of risk [45]. Basieva et al. think that $\cos \left(\theta_{A}(t)\right)$ is an interference factor that affects the subjective judgment of decision-makers, such as risk [46]. Thus, $U_{A_{1}}(t)$ includes the influence of risk factors.

2.4. Subjective Cognition on the Utility of Behavior. Coal miners' subjective cognition on the utility of $A_{2}$ is $U_{A_{2}}(t)=\sum_{k=1}^{2} p_{k}^{\prime \prime}(t) u_{A 2 k}, \quad p_{k}^{\prime \prime}(t)=\left[\sum_{l=1}^{2} \sqrt{y_{l}} \cos \left(\theta_{A}^{\prime}\right.\right.$ $(t))^{\sqrt{y_{k}}}$, and $\theta_{A}^{\prime}(t)=\theta_{l^{A 0}} \longrightarrow k^{A 2}(t)$. When $l=k$, $\varnothing_{l^{A 0} \longrightarrow k^{A 2}}=\varnothing_{l^{A 0} \longrightarrow k^{A 1}}=0$. When $l \neq k, \quad \varnothing_{l^{A 0} \longrightarrow k^{A 2}}=$ $\varnothing_{l^{A 0} \longrightarrow k^{A 1}}=(\pi / 2)$. Thus, $\quad \theta_{A}^{\prime}(t)=\varnothing_{l^{A 0} \longrightarrow k^{A 2}}+\theta_{m k}(t)$ $-\theta_{m l}(t)=\theta_{l^{A 0}} \longrightarrow k^{A 1}(t)=\theta_{A}(t), \quad \cos \left(\theta_{A}^{\prime}(t)\right)=\cos$ $\left(\theta_{A}(t)\right)$, and $p_{k}^{\prime \prime}(t)=p_{k}^{\prime}(t)$. We use the following principle to judge the behavioral preference of coal miners:

$$
\begin{cases}A_{1}>A_{2}, & D_{A}(t)>0, \\ A_{2} \preccurlyeq A_{1}, & D_{A}(t) \leq 0,\end{cases}
$$

where $D_{A}(t)=U_{A_{1}}(t)-U_{A_{2}}(t)$. $U_{A_{1}}(t)$ is the coal miners' subjective cognition on the utility of $A_{1} \cdot U_{A_{2}}(t)$ is coal 


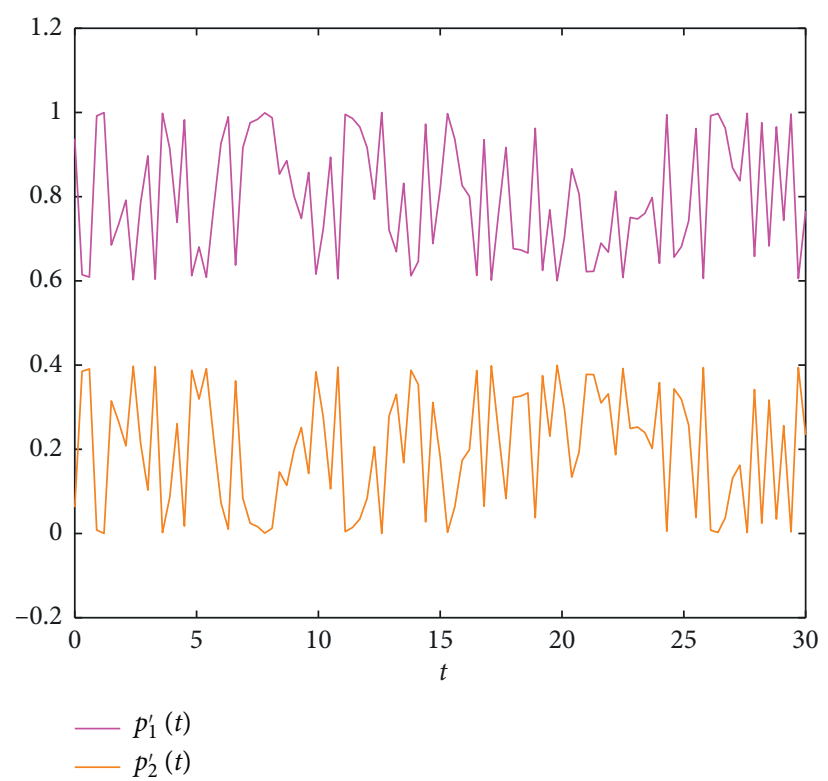

Figure 2: Coal miners' judgments on $y_{1}$ and $y_{2}$ under the influence of random factors.

miners' subjective cognition on the utility of $A_{2}$. When coal miners think that the utility of $A_{1}$ is greater than the utility of $A_{2}$, they will prefer $A_{1}$.

According to the above method, we can construct the operator $D_{B}(t)$ used to compare $B_{1}$ with $B_{2}$ :

$$
D_{B}(t)=U_{B_{1}}(t)-U_{B_{2}}(t)
$$

where

$$
\begin{aligned}
& U_{B_{1}}(t)=\sum_{k=1}^{2} q_{k}(t) u_{B 1 k}, \\
& U_{B_{2}}(t)=\sum_{k=1}^{2} q_{k}(t) u_{B 2 k}, \\
& q_{k}(t)= \begin{cases}0, & q_{k}^{\prime}(t)<0, \\
1, & q_{k}^{\prime}(t)>1, \\
q_{k}^{\prime}(t) & \text { Others. }\end{cases} \\
& q_{1}^{\prime}(t)=x_{1}+\sqrt{x_{1} x_{2}} \cos \left(\theta_{B}(t)\right), \\
& q_{2}^{\prime}(t)=x_{2}-\sqrt{x_{2} x_{1}} \cos \left(\theta_{B}(t)\right),
\end{aligned}
$$

when $D_{B}(t)>0$ and $B_{1}>B_{2}$.

2.5. Behavior Evolution Model. The replicator dynamics equation proposed by Taylor and Jonker is one of the most important models in evolutionary game theory $[49,50]$. It can be used to predict the evolution of population behavior [50]. Suppose that there are two groups, each with two different strategies. The replicator dynamics equation is shown as follows:

$$
\left\{\begin{array}{l}
\frac{\mathrm{d} x}{\mathrm{~d} t}=x\left(u_{1}-\bar{u}\right)=x(1-x)\left(u_{1}-u_{2}\right), \\
\frac{\mathrm{d} y}{\mathrm{~d} t}=y\left(v_{1}-\bar{v}\right)=y(1-y)\left(v_{1}-v_{2}\right),
\end{array}\right.
$$

where $u_{1}$ and $u_{2}$ are the utility of individuals in the first group when they choose strategy 1 and strategy 2 , respectively. $\bar{u}$ is the average utility of the first group. $x$ is the proportion of choosing strategy $1 . v_{1}$ and $v_{2}$ are the utility of individuals in the second group when they choose strategy 3 and strategy 4 , respectively. $\bar{v}$ is the average utility of the second group. $y$ is the proportion of those choosing strategy 3.

We assume that the game between coal miners and supervisors is a repeated game with a random pair for the big group consisting of members whose learning speeds are relatively slow. We assume that coal miners and supervisors choose behaviors according to their subjective judgment. Their subjective cognition on the utility of each behavior is $U_{A_{1}}(t)=u_{A 11} p_{1}(t)+u_{A 12} p_{2}(t), U_{A 2}(t)=u_{A 21} p_{1}(t)+u_{A 22}$ $p_{2}(t), \quad U_{B 1}(t)=u_{B 11} q_{1}(t)+u_{B 12} q_{2}(t), \quad$ and $\quad U_{B 2}(t)=$ $u_{B 21} q_{1}(t)+u_{B 22} q_{2}(t)$. According to the replication dynamic equation, the following behavior evolution model can be established:

$$
\left\{\begin{array}{l}
\frac{\mathrm{d} x}{\mathrm{~d} t}=x(1-x) D_{A}(t), \\
\frac{\mathrm{d} y}{\mathrm{~d} t}=y(1-y) D_{B}(t),
\end{array}\right.
$$

where 


$$
\begin{aligned}
& x=x_{1}, \\
& y=y_{1}, \\
& D_{A}(t)=U_{A_{1}}(t)-U_{A_{2}}(t) \text {, } \\
& D_{B}(t)=U_{B_{1}}(t)-U_{B_{2}}(t) \text {, } \\
& p_{1}(t)= \begin{cases}0, & y+\sqrt{y(1-y)} \cos \left(\theta_{A}(t)\right)<0, \\
1, & y+\sqrt{y(1-y)} \cos \left(\theta_{A}(t)\right)>1, \\
y+\sqrt{y(1-y)} \cos \left(\theta_{A}(t)\right), & \text { Others, }\end{cases} \\
& q_{1}(t)= \begin{cases}0, & x+\sqrt{x(1-x)} \cos \left(\theta_{B}(t)\right)<0, \\
1, & x+\sqrt{x(1-x)} \cos \left(\theta_{B}(t)\right)>1, \\
x+\sqrt{x(1-x)} \cos \left(\theta_{B}(t)\right), & \text { Others. }\end{cases}
\end{aligned}
$$

\section{Behavior Choice of Game Parties}

3.1. Behavior Choice at Time t. The relevant assumptions in the game between coal miners and supervisors are as follows:

Taking $u_{0}=7, I=3, f=6, b=1, c_{0}=3$, and $l=5$ as an example, we analyze the behavior choice of coal miners and supervisors in different situations. Substituting the above parameters into $D_{A}(t)$ and $D_{B}(t)$, we can conclude $D_{A}(t)=$ $-4 p_{1}(t)+3\left[1-p_{1}(t)\right]$ and $D_{B}(t)=2 q_{1}(t)-3\left[1-q_{1}(t)\right]$. When $D_{A}(t)>0$, coal miners will prefer the operation in violation of the rules. When $D_{B}(t)>0$, supervisors will prefer supervision. With the change in $\theta_{A}(t), D_{A}(t)$ is shown in Figure $3(\mathrm{a})$. With the change in $\theta_{B}(t), D_{B}(t)$ is shown in Figure 3(b).

According to Figure 3(a), whether coal miners choose to operate in violation of rules depends on the probability of supervising and $\cos \left(\theta_{A}(t)\right)$. When $\cos \left(\theta_{A}(t)\right)<0$, the coal miners will overrate the utility of the operation in violation of the rules. When $\theta_{A}(t)$ remains constant, the smaller the probability of supervising, the more likely it is that coal miners will operate in violation of the rules. When $y$ remains constant, coal miners will be more likely to operate in violation of the rules if they overrate the utility of the operation in violation of the rules seriously. According to Figure 3(b), when $x$ and $\cos \left(\theta_{B}(t)\right)$ are relatively large, supervisors will be more likely to supervise.

3.2. Behavior Choice with the Change in $t$. The behavior evolution process is discussed in the two following situations. The first situation is complete cognition. In the condition of complete cognition, $\cos \left(\theta_{A}(t)\right)$ and $\cos \left(\theta_{B}(t)\right)$ are 0 . The behavior evolution process is shown in Figure 4. $x$ is the probability of choosing $A_{1}$, which is also the violation probability. $y$ is the probability of choosing $B_{1}$, which is also the supervision probability.

According to Figure 4, different original states will have different evolution processes. The evolution process shown in Figure 4 is not stable. As can be seen from Figure 4, an increase in the violation probability will increase the supervision probability. When the probability of supervision increases to a certain extent, the violation probability will change from the increase to decrease. When the violation probability decreases to a certain extent, the probability of supervision will decrease. When the probability of supervision decreases to a certain extent, the violation probability will change from the decrease to increase. In Figure $4(\mathrm{a})$, when $t=15, x=0.77$ and $y=0.42$. At this time, $x$ will change from the increase to decrease. When $t=16, x=0.58$ and $y=0.58$. At this time, $y$ will change from the increase to decrease. When $t=17.4, x=0.42$ and $y=0.42$. At this time, $x$ will change from the decrease to increase.

The second situation is fluctuation in the game parties' cognitive state. It can be assumed that $\cos \left(\theta_{A}(t)\right)$ fluctuates in $\left[f_{A}^{0}, f_{A}^{1}\right]$ and $\cos \left(\theta_{B}(t)\right)$ fluctuates in $\left[f_{B}^{0}, f_{B}^{1}\right]$. We use $\cos \left(\theta_{A}(t)\right)=f_{A}^{0}+\left(f_{A}^{1}-f_{A}^{0}\right)|\xi(t)|$ and $\cos \left(\theta_{B}(t)\right)=f_{B}^{0}+$ $\left(f_{B}^{1}-f_{B}^{0}\right)|\xi(t)|$ to describe the fluctuation in $\cos \left(\theta_{A}(t)\right)$ and $\cos \left(\theta_{B}(t)\right)$ in $\left[f_{A}^{0}, f_{A}^{1}\right]$ and $\left[f_{B}^{0}, f_{B}^{1}\right]$, respectively, where $\xi(t)=\cos (\omega t+U)$, and $U$ obeys the uniform distribution of $[0,2 \pi]$. Through specific examples, we adopt the emulation mode to analyze the impact of the fluctuation in the game parties' cognitive state on the evolution process and the stability of the balance points. If $\left(x^{*}, y^{*}\right)$ satisfies $x^{*}(1-$ $\left.x^{*}\right) D_{A}(t)=0$ and $y^{*}\left(1-y^{*}\right) D_{B}(t)=0,\left(x^{*}, y^{*}\right)$ is the balance point of (19). During the analysis, we use $\sigma * \cos \left(\theta_{A}(t)\right)$ to analyze the behavior evolution process after the amplification of the fluctuation.

We take $x_{t=0}=0.5$ and $y_{t=0}=0.3$ as the original state of the evolution system and then analyze the impact of the fluctuation in the game parties' cognitive state on the behavior evolution process. When $f_{A}^{0}<0<f_{A}^{1}$ and $f_{B}^{0}<0<f_{B}^{1}$, the behavior evolution process is shown in Figure 5, where $\sigma=2$. According to Figure 5, if the fluctuation scope of $\cos \left(\theta_{A}(t)\right)$ and $\cos \left(\theta_{B}(t)\right)$ is bigger, the fluctuation scope of $x$ and $y$ will be bigger. If the fluctuation scope of $\cos \left(\theta_{A}(t)\right)$ and $\cos \left(\theta_{B}(t)\right)$ is smaller, the behavior evolution process will be more similar to the behavior evolution process in the condition of complete cognition.

When $f_{A}^{1}<0, f_{B}^{1}<0$ or $f_{A}^{0}>0, f_{B}^{0}>0$, the behavior evolution process is shown in Figure 6. In Figure 6, $x_{0}, y_{0}$ is the behavior evolution process in the condition of complete cognition. Figure $6(\mathrm{a})$ is the behavior evolution process when $\cos \left(\theta_{A}(t)\right)<0$ and $\cos \left(\theta_{B}(t)\right)<0$. Figure $6(\mathrm{~b})$ is the behavior evolution process when $\cos \left(\theta_{A}(t)\right)>0$ and 


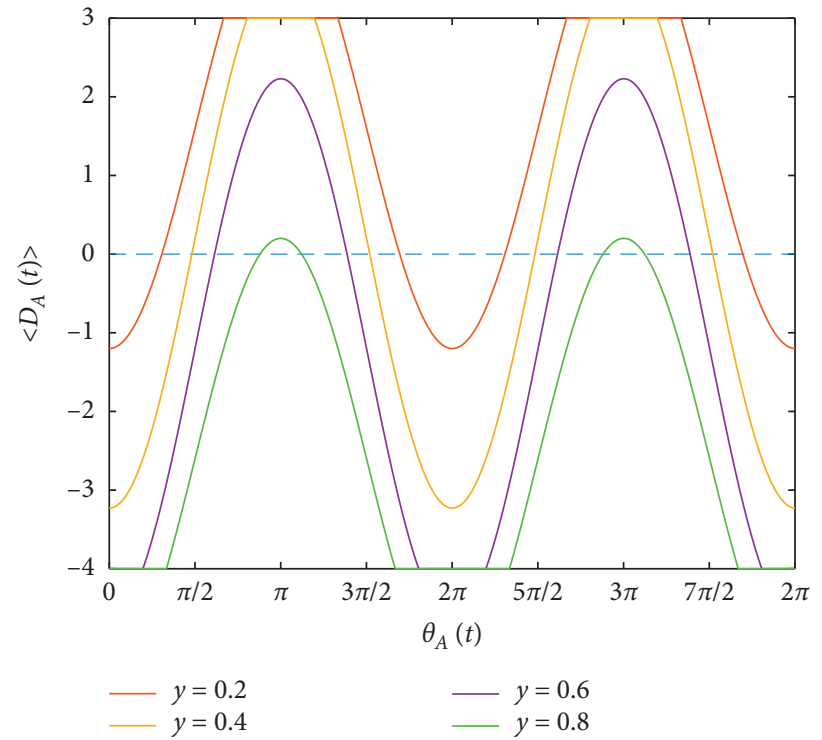

(a)

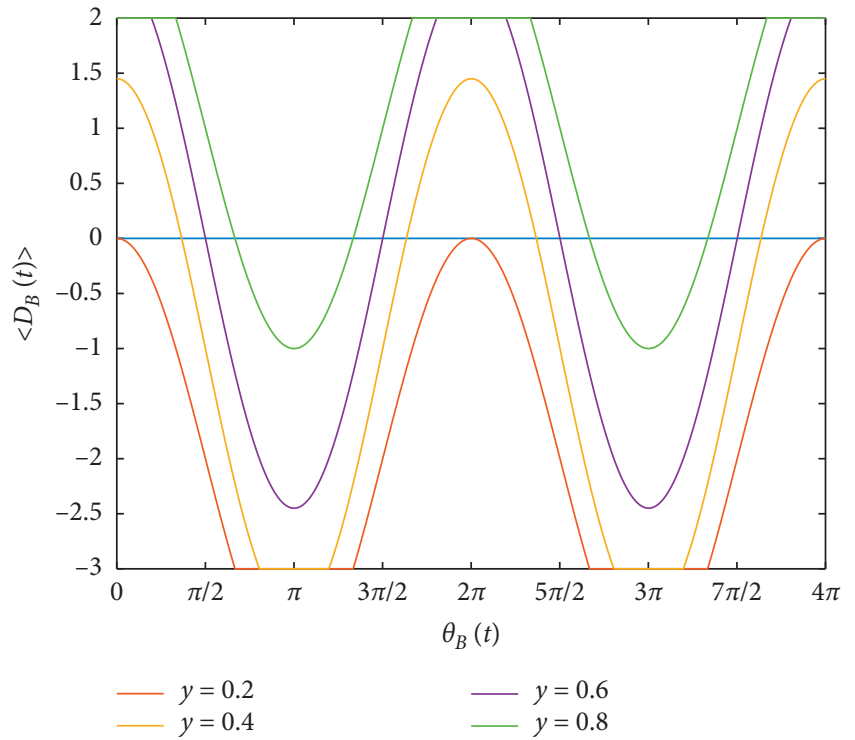

(b)

FIGURE 3: The change process of preference of coal miners and supervisors. (a) The change process of coal miners' preference with the change in $\theta_{A}(t)$. (b) The change process of supervisors' preference with the change in $\theta_{B}(t)$.

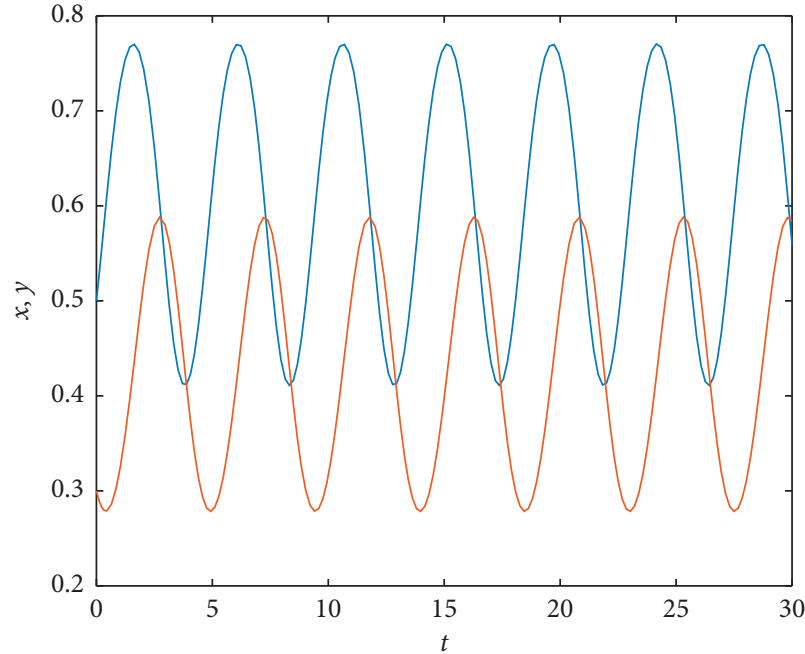

$-x$

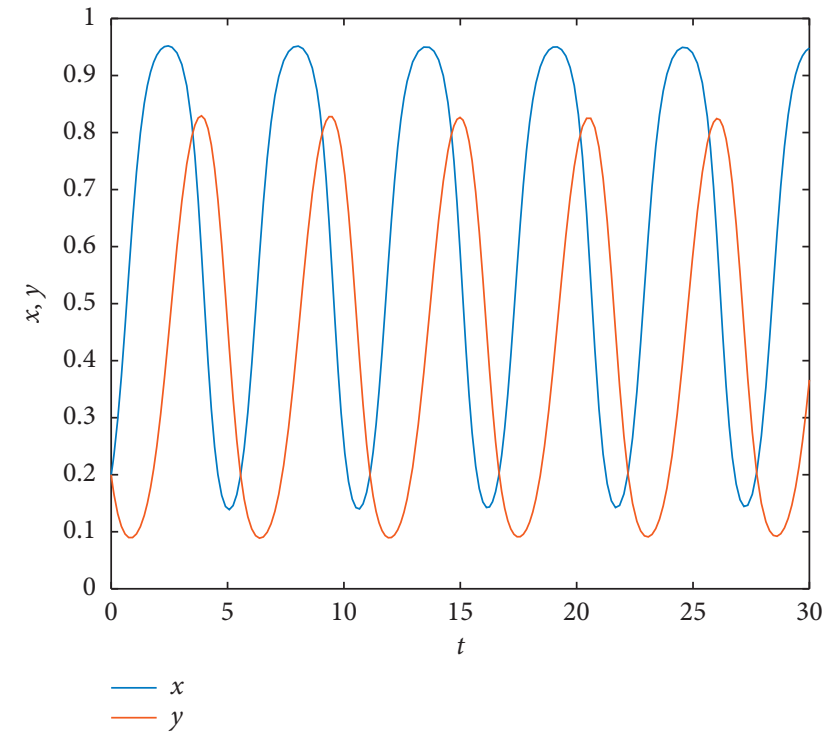

(b)

Figure 4: The evolution process in the condition of complete cognition. (a) The evolution process when the initial state is $x_{0}=0.5$ and $y_{0}=0.3$. (b) The evolution process when the initial state is $x_{0}=0.2$ and $y_{0}=0.2$.

$\cos \left(\theta_{B}(t)\right)>0 . \cos \left(\theta_{A}(t)\right)<0$ represents that coal miners overrate the utility of $A_{1}$. According to Figure 6(a), coal miners are more likely to operate in violation of the rules when they overrate the utility of $A_{1}$. According to Figure 6(b), coal miners are more likely to operate according to the rules when they underestimate the utility of $A_{1}$. The fluctuation in the game parties' cognitive state can affect the process of behavior evolution.

\section{Behavior Control Strategy}

For controlling the operation in violation of the rules, it is necessary to let $(0,0)$ or $(0,1)$ become the stable point of the evolution system. We first assume that coal miners and supervisors can achieve complete cognition and then use the Jacobi matrix to analyze the stability of the balance points of the evolution system [50]. Finally, we analyze whether the 


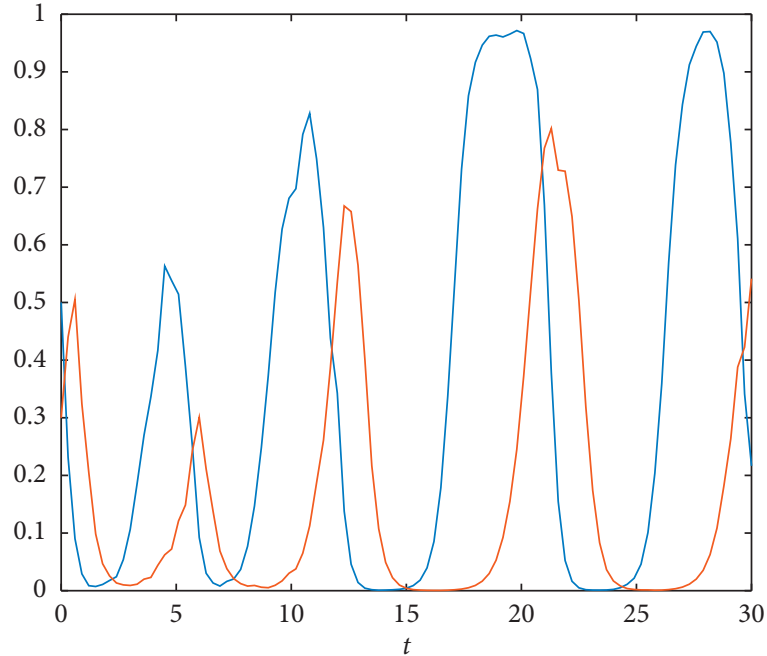

$-x$

(a)
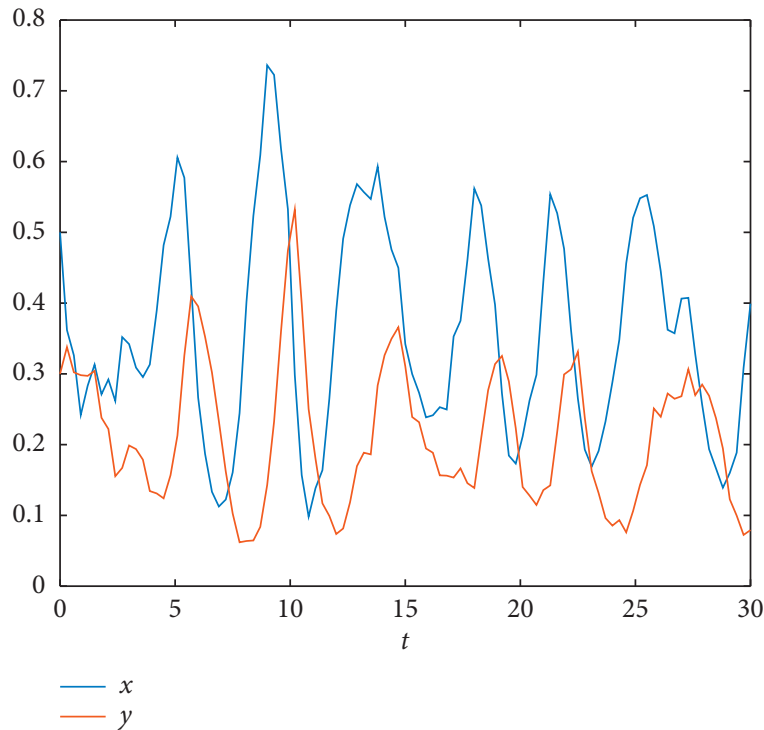
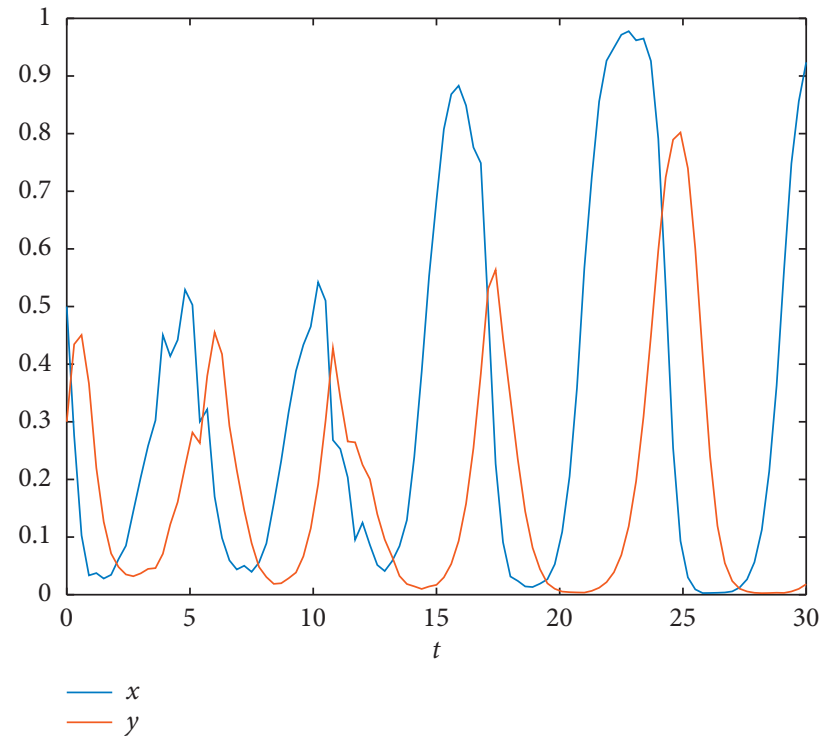

(b)
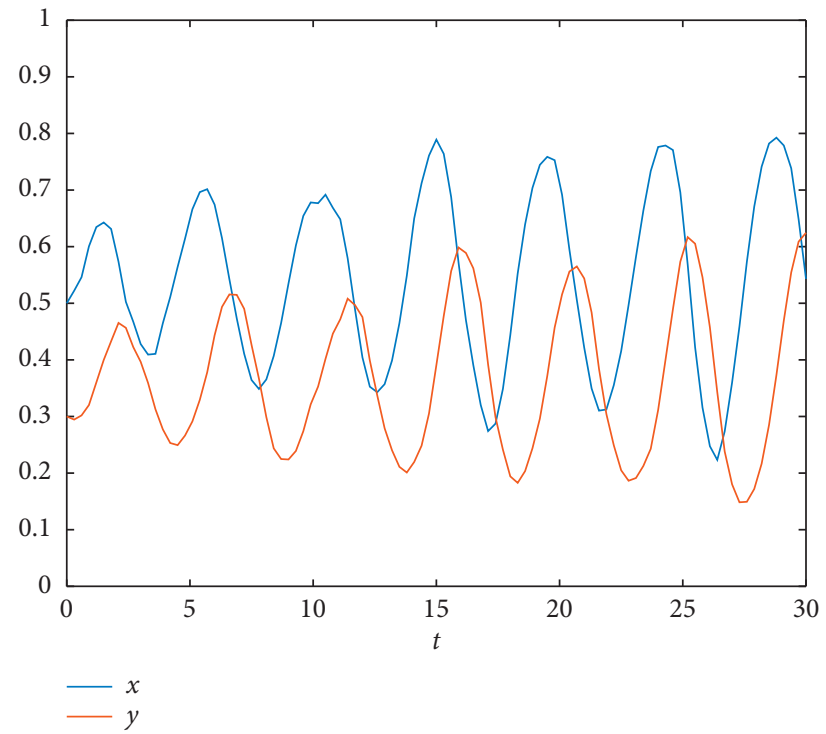

(d)

FIGURE 5: The evolution processes when game parties' cognitive state fluctuates in different ranges. (a) The behavior evolution process when the fluctuation range is $\left[f_{A}^{0}, f_{A}^{1}\right]=[-1,1]$ and $\left[f_{B}^{0}, f_{B}^{1}\right]=[-1,1]$. (b) The behavior evolution process when the fluctuation range is $\left[f_{A}^{0}, f_{A}^{1}\right]=[-0.7,0.7]$ and $\left[f_{B}^{0}, f_{B}^{1}\right]=[-0.7,0.7]$. (c) The behavior evolution process when the fluctuation range is $\left[f_{A}^{0}, f_{A}^{1}\right]=[-0.4,0.4]$ and $\left[f_{B}^{0}, f_{B}^{1}\right]=[-0.4,0.4]$. (d) The behavior evolution process when the fluctuation range is $\left[f_{A}^{0}, f_{A}^{1}\right]=[-0.1,0.1]$ and $\left[f_{B}^{0}, f_{B}^{1}\right]=[-0.1,0.1]$.

fluctuation of game parties' cognitive state affects the control effect of the control strategy. Label $(\mathrm{d} x / \mathrm{d} t)$ and $(\mathrm{d} y / \mathrm{d} t)$ as $f(x, y)$ and $(x, y)$, respectively. The Jacobi matrix is shown as follows:

$$
J=\left(\begin{array}{ll}
f_{x}^{\prime}(x, y) & f_{y}^{\prime}(x, y) \\
g_{x}^{\prime}(x, y) & g_{y}^{\prime}(x, y)
\end{array}\right),
$$

$$
\begin{aligned}
& f_{x}^{\prime}(x, y)=(1-2 x)\left[\left(u_{A 11}-u_{A 21}\right) y+\left(u_{A 12}-u_{A 22}\right)(1-y)\right], \\
& f_{y}^{\prime}(x, y)=x(1-x)\left[\left(u_{A 11}-u_{A 21}\right)-\left(u_{A 12}-u_{A 22}\right)\right], \\
& g_{y}^{\prime}(x, y)=(1-2 y)\left[\left(u_{B 11}-u_{B 21}\right) x+\left(u_{B 12}-u_{B 22}\right)(1-x)\right], \\
& g_{x}^{\prime}(x, y)=y(1-y)\left[\left(u_{B 11}-u_{B 21}\right)-\left(u_{B 12}-u_{B 22}\right)\right],
\end{aligned}
$$

where 


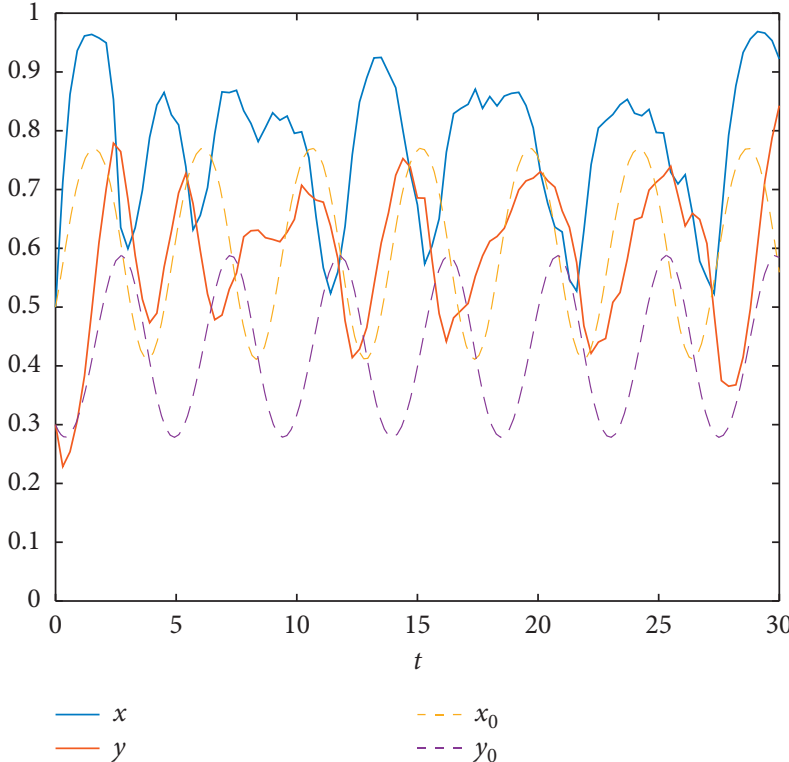

(a)

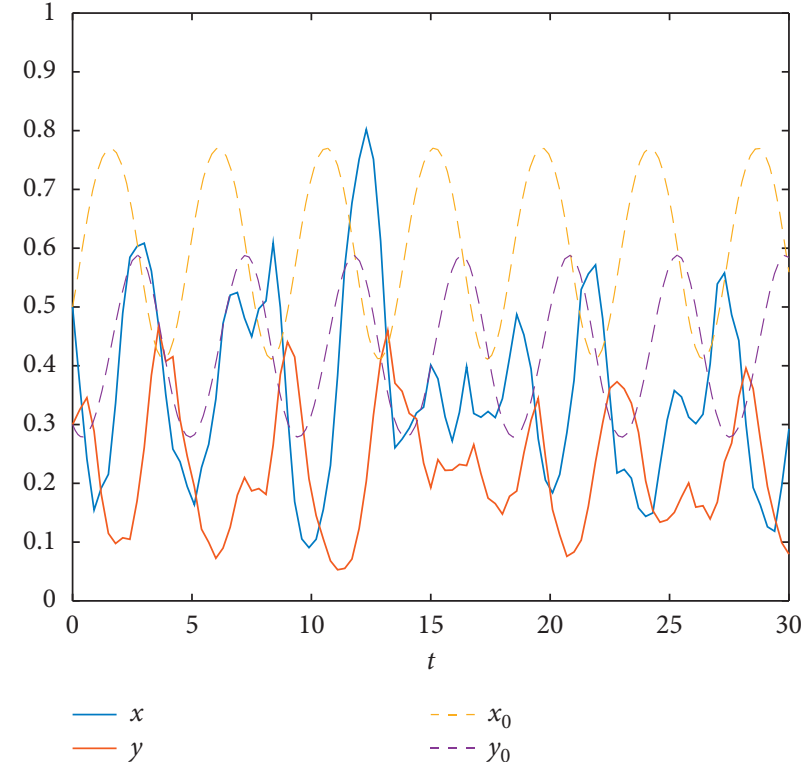

(b)

Figure 6: Comparison of evolution processes under complete cognition and cognitive state fluctuation. (a) The evolution process under complete cognition and the evolution process when $\left[f_{A}^{0}, f_{A}^{1}\right]=[-0.8,-0.6]$ and $\left[f_{B}^{0}, f_{B}^{1}\right]=[-0.8,-0.6]$. (b) The evolution process under complete cognition and the evolution process when $\left[f_{A}^{0}, f_{A}^{1}\right]=[0.6,0.8]$ and $\left[f_{B}^{0}, f_{B}^{1}\right]=[0.6,0.8]$.

for the balance point $\left(x_{0}, y_{0}\right)$, when $\operatorname{det} J=f_{x}^{\prime}\left(x_{0}, y_{0}\right) g_{y}^{\prime}\left(x_{0}, y_{0}\right)-f_{y}^{\prime}\left(x_{0}, y_{0}\right) g_{x}^{\prime}\left(x_{0}, y_{0}\right)>0$, $\operatorname{tr} J=f_{x}^{\prime}\left(x_{0}, y_{0}\right)+g_{y}^{\prime}\left(x_{0}, y_{0}\right)<0,\left(x_{0}, y_{0}\right)$ is a stable point of evolution.

The condition where $(0,0)$ is a stable point has $\left(u_{A 12}-\right.$ $\left.u_{A 22}\right)\left(u_{B 12}-u_{B 22}\right)>0$ and $\left(u_{A 12}-u_{A 22}\right)+\left(u_{B 12}-u_{B 22}\right)<0$. The condition where $(0,1)$ is a stable point has $\left(u_{A 11}-\right.$ $\left.u_{A 21}\right)\left(u_{B 12}-u_{B 22}\right)<0$ and $\left(u_{A 11}-u_{A 21}\right)-\left(u_{B 12}-u_{B 22}\right)<0$.

Because $\left(u_{A 12}-u_{A 22}\right)\left(u_{B 12}-u_{B 22}\right)=-I c_{0}<0,(0,0)$ is an unstable point. When $I-f-b>0$, $\left(u_{A 11}-u_{A 21}\right)-\left(u_{B 12}-u_{B 22}\right)=I-f-b+c_{0}>0$. When $I-f-b<0$,

$\left(u_{A 11}-u_{A 21}\right)\left(u_{B 12}-u_{B 22}\right)=(I-f-b)\left(-c_{0}\right)>0 . \quad(0,1)$ is also an unstable point. Therefore, $(0,0)$ or $(0,1)$ cannot become a stable point only by "punishing coal miners for operating in violation of rules" and "awarding coal miners for operating according to the rules."

To make $(0,0)$ or $(0,1)$ become a stable point, we introduce the "punishing supervisors for not supervising." The combined strategy consisting of "punishing coal miners for operating in violation of rules," "awarding coal miners for operating according to the rules," and "punishing supervisors for not supervising" is adopted to control the operation in violation of the rules. It can be assumed that the punishment that the supervisor will suffer if he does not supervise is $f^{\prime} \cdot u_{B 12}-u_{B 22}=f_{l}-c_{0}$.

When $f \prime-c_{0}>0, \quad\left(u_{A 12}-u_{A 22}\right)+\left(u_{B 12}-u_{B 22}\right)=I+$ $f \prime-c_{0}>0$. When $f \prime-c_{0}<0,\left(u_{A 12}-u_{A 22}\right)\left(u_{B 12}-u_{B 22}\right)=$ $I\left(f,-c_{0}\right)<0$. Hence, the introduction of the strategy of "punishing supervisors for not supervising" cannot make
$(0,0)$ become a stable point. When $\left(u_{A 11}-u_{A 21}\right)\left(u_{B 12}-\right.$ $\left.u_{B 22}\right)=(I-f-b)\left(f I-c_{0}\right)<0$ and $\left(u_{A 11}-u_{A 21}\right)-$ $\left(u_{B 12}-u_{B 22}\right)=(I-f-b)-\left(f \prime-c_{0}\right)<0,(0,1)$ is a stable point. At this point, the operation in violation of the rules on the part of the coal miner can be controlled. For this case, $I-f-b=-4<0$ and $c_{0}=3$. Thus, when $f \prime>3,(0,1)$ is a stable point.

According to the impact of the fluctuation in the game parties' cognitive state on behavior evolution, coal miners are more likely to choose to operate in violation of the rules when they underestimate $y$ and the supervisors underestimate $x$. We let $\left[f_{A}^{0}, f_{A}^{1}\right]=[-1,-0.6]$ and $\left[f_{B}^{0}, f_{B}^{1}\right]=[-1,-0.6]$ and use $\sigma * \cos \left(\theta_{A}(t)\right)$ to amplify the coal miners' underestimated degree of $y$ and use $\sigma * \cos \left(\theta_{B}(t)\right)$ to amplify the supervisors' underestimated degree of $x$. When $f_{l}<c_{0}$, the behavior evolution process is shown as in Figure $7(\mathrm{a})$. When $f \prime>c_{0}$ and $\sigma$ have different values, the behavior evolution process is shown in Figures $7(\mathrm{~b})-7(\mathrm{~d})$. In Figure $6, x_{0}, y_{0}$ is the evolution process in the condition of complete cognition.

According to Figure 7, the fluctuation in the game parties' cognitive state can affect the time when the final effect of the control strategy is achieved. By using $\sigma * \cos \left(\theta_{A}(t)\right)$ and $\sigma * \cos \left(\theta_{B}(t)\right)$ to let the cognitive state have a fluctuation in different scopes, the system will finally stabilize in the point of $(0,1)$. This indicates that $\cos \left(\theta_{A}(t)\right)$ and $\cos \left(\theta_{B}(t)\right)$ cannot affect the stability of $(0,1)$. The fluctuation in the game parties' cognitive state does not affect the eventual effect of the control strategy, but some fluctuations will extend the time when the final effect is achieved. 


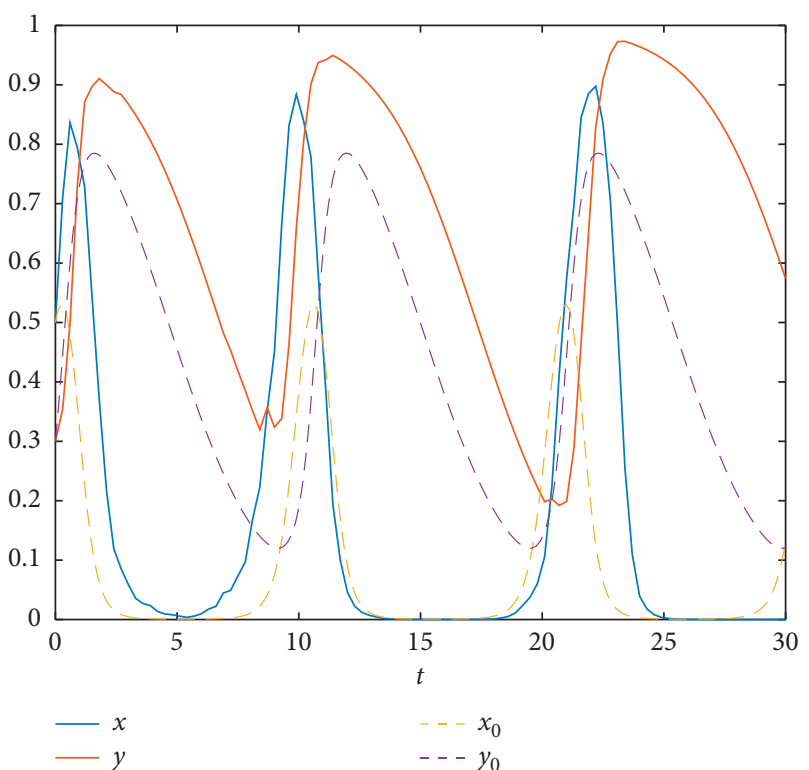

(a)

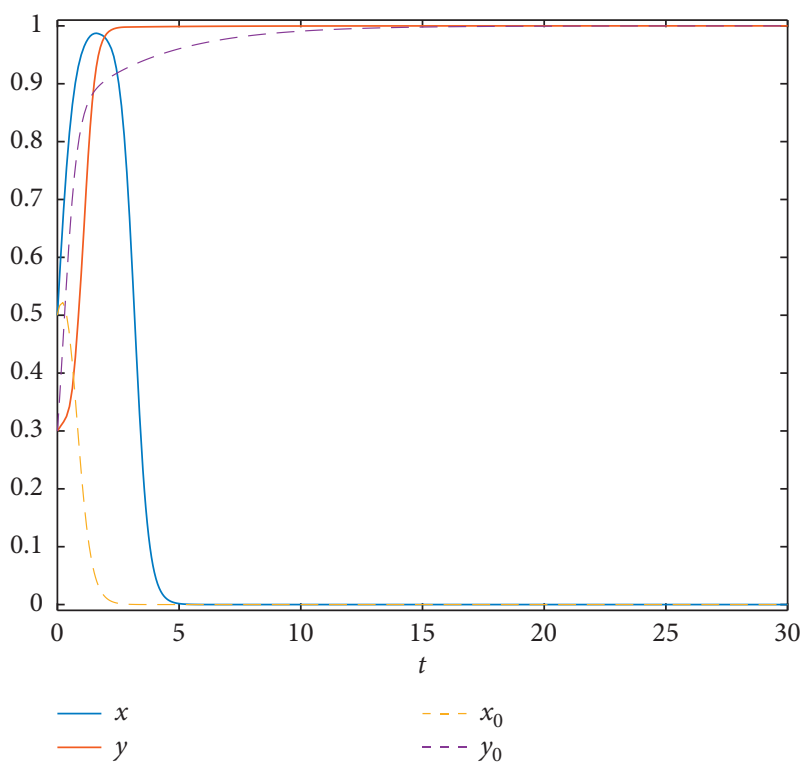

(c)

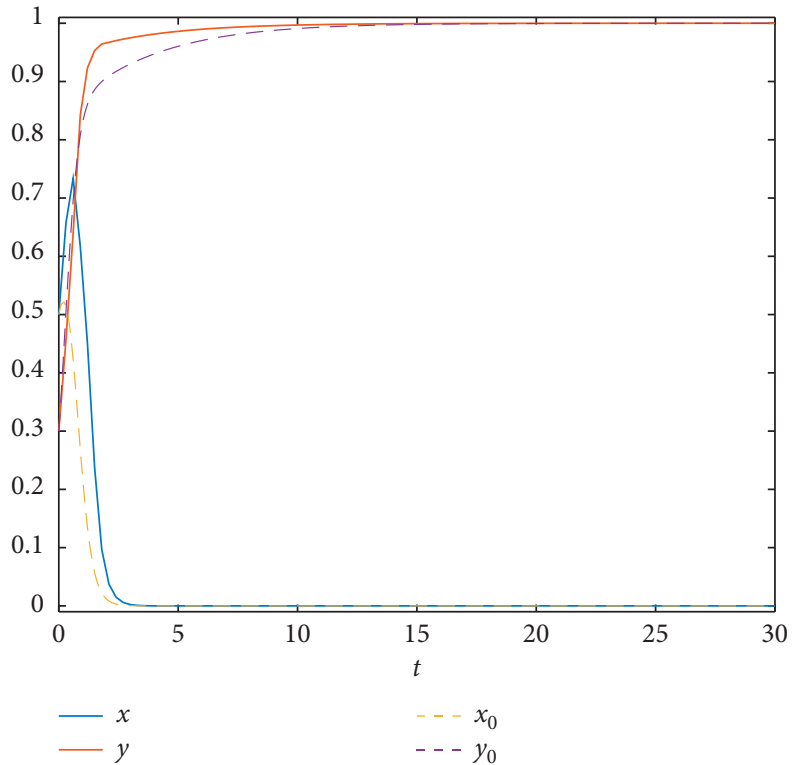

(b)

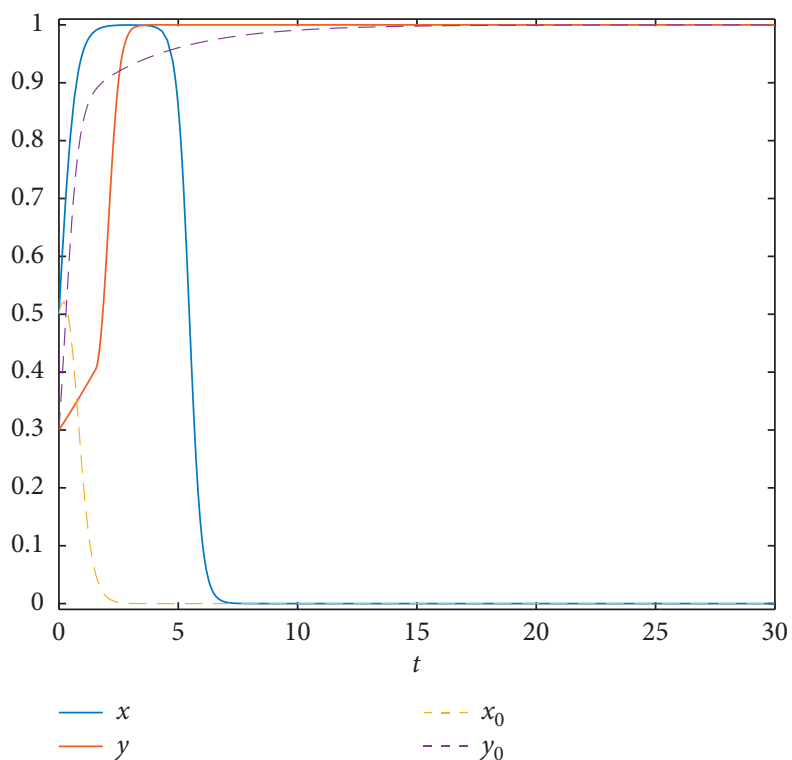

(d)

Figure 7: The evolution processes under different levels of punishment and cognitive fluctuations. (a) The behavior evolution process when $f \prime=2.5$ and $\sigma=1$. (b) The behavior evolution process when $f \prime=3.5$ and $\sigma=1$. (c) The behavior evolution process when $f \prime=3.5$ and $\sigma=2$. (d) The behavior evolution process when $f \prime=3.5$ and $\sigma=10$.

\section{Discussion and Conclusions}

The traditional game model is based on the assumption that both sides of the game are completely rational, which cannot reflect the subjective cognition of game parties on the utility of behavior. In the game between coal miners and supervisors, their cognitive ability is limited. Under the influence of random factors, the cognition of coal miners on the utility of the operation in violation of the rules is biased, sometimes overestimating its utility and sometimes underestimating its utility. When coal miners think that the utility of the operation in violation of the rules is higher than that of operations according to the rules, they will prefer the operation in violation of the rules. The subjective cognition and preference of behaviors can be described by (14) and (15). Actually, the cognition and preference of coal miners to the operation in violation of the rules are not fixed. Under the influence of random factors, the cognitive state and behavior preference of coal miners have stochastic volatility. (14) and (15) can describe this stochastic volatility. The description method of subjective cognition can be applied to the behavior analysis of other occupational groups.

In the game process of coal miners and supervisors, the game parties will continue to learn. Through learning, their 
cognition will change. The change in cognition will affect their behavior choice. Based on the evolutionary game theory, the behavior evolution model, which is shown in (18), can describe the behavior evolution process of coal miners and supervisors. Compared with previous evolutionary game models, (18) embeds a stochastic process. This provides a reference for the analysis of the behavior evolution process under the interference of stochastic factors. The probability of supervisors' choice of supervision and the cognition of coal miners on the probability of supervision will affect the cognition of coal miners on the utility of operation in violation of the rules. When the probability of supervision is low and the coal miners overrate the utility of the operation in violation of the rules, the coal miners will tend to choose the operation in violation of the rules, as is shown in Figure 3(a).

The behaviors of coal miners and supervisors influence each other. When the fluctuation in the cognitive state is not considered and the probability of operation in violation of the rules is low, supervisors will tend to choose not to supervise. This will reduce the probability of supervision. The decrease in the probability of supervision will make coal miners tend to choose the operation in violation of the rules. The increase in the probability of operation in violation of the rules will make supervisors tend to choose supervision. The behavior relationship between coal miners and supervisors is shown in Figure 4. When considering the fluctuation of cognitive state, the behavior evolution process of coal miners and supervisors will become complex, as is shown in Figure 5.

Different coal miners have different cognitions. No matter how the cognitive state fluctuates, some coal miners may always overestimate the utility of the operation in violation of the rules. Compared with the coal miners without cognitive bias, they are more inclined to choose the operation in violation of the rules. This also makes supervisors more inclined to choose supervision. The behavior evolution process in this case is shown in Figure 6(a). Some coal miners may always underestimate the effectiveness of the operation in violation of the rules. Compared with the coal miners without cognitive bias, they prefer to operate according to the rules. This makes supervisors more inclined to choose not to supervise. The behavior evolution process in this case is shown in Figure 6(b).

It can be seen from Figures 4-6 that managing the behavior of the coal miners only cannot completely control the operation in violation of the rules. It is also necessary to manage the behavior of supervisors. The combined strategy consisting of "punishing coal miners for operating in violation of rules," "awarding coal miners for operating according to the rules," and "punishing supervisors for not supervising" can make coal miners choose the operation according to rules and supervisors choose supervision. The fluctuation of cognitive states of coal miners and supervisors will not affect the final effect of this strategy.

The description method of subjective cognition can describe the cognitive state, where coal miners and supervisors overestimate or underestimate the utility of behaviors under the influence of random factors. The behavior evolution model with random variables can describe the dynamic evolution process of the behavior choice of coal miners and supervisors. The behavior evolution model can be used to analyze the behavior relationship between coal miners and supervisors under the conditions of random fluctuations in cognitive state. In coal mine production, the behavior of coal miners is also affected by the behavior of their colleagues and production commanders. In the future, we will establish a behavior evolution model that includes multiple game players.

\section{Data Availability}

All data used to support the findings of this study are included within the article.

\section{Conflicts of Interest}

The authors declare that they have no conflicts of interest.

\section{Acknowledgments}

This work was supported by the National Natural Science Foundation of China (Grant no. 51574157): Research on Multilateral Game and Control Strategies in Coal Mine Safety System.

\section{References}

[1] C. Wang, J. Wang, X. Wang, H. Yu, L. Bai, and Q. Sun, "Exploring the impacts of factors contributing to unsafe behavior of coal miners," Safety Science, vol. 115, pp. 339-348, 2019.

[2] R. Tong, Y. Zhang, P. Cui et al., "Characteristic analysis of unsafe behavior by coal miners: multi-dimensional description of the pan-scene data," International Journal of Environmental Research and Public Health, vol. 15, no. 8, Article ID 1608, 2018.

[3] K. Yu, Q. Cao, C. Xie, N. Qu, and L. Zhou, "Analysis of intervention strategies for coal miners' unsafe behaviors based on analytic network process and system dynamics," Safety Science, vol. 118, pp. 145-157, 2019.

[4] Q. Cao, K. Yu, L. Zhou, L. Wang, and C. Li, "In-depth research on qualitative simulation of coal miners' group safety behaviors," Safety Science, vol. 113, pp. 210-232, 2019.

[5] G. Fu, X. Xie, Q. Jia, W. Tong, and Y. Ge, "Accidents analysis and prevention of coal and gas outburst: understanding human errors in accidents," Process Safety and Environmental Protection, vol. 134, pp. 1-23, 2020.

[6] H. Yu, H. Chen, and R. Long, "Mental fatigue, cognitive bias and safety paradox in Chinese coal mines," Resources Policy, vol. 52, pp. 165-172, 2017.

[7] M. Mendl, "Performing under pressure: stress and cognitive function," Applied Animal Behaviour Science, vol. 65, no. 3, pp. 221-244, 1999.

[8] H. Lieberman, W. Tharion, B. Shukitt-Hale, K. Speckman, and R. Tulley, "Effects of caffeine, sleep loss, and stress on cognitive performance and mood during U.S. Navy SEAL training," Psychopharmacology, vol. 164, no. 3, pp. 250-261, 2002.

[9] K. Starcke, O. T. Wolf, H. J. Markowitsch, and M. Brand, "Anticipatory stress influences decision making under explicit 
risk conditions," Behavioral Neuroscience, vol. 122, no. 6, pp. 1352-1360, 2008.

[10] V. Capraro and M. Perc, "Mathematical foundations of moral preferences," Journal of the Royal Society Interface, vol. 18, no. 175, Article ID 20200880, 2021.

[11] Z. Wang, M. Jusup, H. Guo et al., "Communicating sentiment and outlook reverses inaction against collective risks," Proceedings of the National Academy of Sciences, vol. 117, no. 30, pp. 17650-17655, 2020.

[12] D. G. Goldstein and G. Gigerenzer, "Models of ecological rationality: the recognition heuristic," Psychological Review, vol. 109, no. 1, pp. 75-90, 2002.

[13] C. A. Hartley and E. A. Phelps, "Anxiety and decisionmaking," Biological Psychiatry, vol. 72, no. 2, pp. 113-118, 2012.

[14] C. Moreira and A. Wichert, "Quantum probabilistic models revisited: the case of disjunction effects in cognition," Frontiers in Physics, vol. 4, pp. 1-26, 2016.

[15] C. Moreira and A. Wichert, "The synchronicity principle under quantum probabilistic inferences," NeuroQuantology, vol. 13, no. 1, pp. 111-133, 2015.

[16] C. Moreira and A. Wichert, "Quantum-like Bayesian networks for modeling decision-making," Frontiers in Psychology, vol. 7, pp. 1-20, 2016.

[17] C. Moreira and A. Wichert, "Exploring the relations between quantum-Like Bayesian networks and decision-making tasks with regard to face stimuli," Journal of Mathematical Psychology, vol. 78, pp. 86-95, 2017.

[18] C. Moreira and A. Wichert, "Are quantum-like Bayesian networks more powerful than classical Bayesian networks?" Journal of Mathematical Psychology, vol. 82, pp. 73-83, 2018.

[19] C. Moreira, P. Tiwari, H. M. Pandey, P. Bruza, and A. Wichert, "Quantum-like influence diagrams for decision-making," Neural Networks, vol. 132, pp. 190-210, 2020.

[20] N. Bohr, "The quantum postulate and the recent development of atomic theory," Nature, vol. 121, no. 3050, pp. 580-590, 1928.

[21] J. R. Busemeyer and Z. Wang, "What is quantum cognition, and how is it applied to psychology?" Current Directions in Psychological Science, vol. 24, no. 3, pp. 163-169, 2015.

[22] M. Ashtiani and M. A. Azgomi, "A survey of quantum-like approaches to decision making and cognition," Mathematical Social Sciences, vol. 75, pp. 49-80, 2015.

[23] F. Vaio, "The quantum-like approach to modeling classical rationality violations: an introduction," Mind \& Society, vol. 18, no. 1, pp. 105-123, 2019.

[24] R. Lu, X. Wang, and D. Li, "A fractional supervision game model of multiple stakeholders and numerical simulation," Mathematical Problems in Engineering, vol. 2017, Article ID 9123624, 2017.

[25] R. Lu, X. Wang, H. Yu, and D. Li, "Multiparty evolutionary game model in coal mine safety management and its application," Complexity, vol. 2018, Article ID 6987427, 2018.

[26] X. Wang, R. Lu, H. Yu, and D. Li, "Stability of the evolutionary game system and control strategies of behavior instability in coal mine safety management," Complexity, vol. 2019, Article ID 6987427, 2019.

[27] B. Li, X. Wang, R. Lu, H. Yu, and D. Li, "Evolutionary stability analysis of the coal mine safety management system governed by delay and impulsive differential equations," Complexity, vol. 2020, Article ID 6210465, 2020.

[28] Q. Liu, X. Li, and X. Meng, "Effectiveness research on the multi-player evolutionary game of coal-mine safety regulation in China based on system dynamics," Safety Science, vol. 111, pp. 224-233, 2019.

[29] Q. Liu, X. Li, and M. Hassall, "Evolutionary game analysis and stability control scenarios of coal mine safety inspection system in China based on system dynamics," Safety Science, vol. 80, pp. 13-22, 2015.

[30] L. Ma, Q. Liu, Z. Qiu, and Y. Peng, "Evolutionary game analysis of state inspection behaviour for coal enterprise safety based on system dynamics," Sustainable Computing: Informatics and Systems, vol. 28, Article ID 100430, 2020.

[31] K. Yu, L. Zhou, Q. Cao, and Z. Li, "Evolutionary game research on symmetry of workers' behavior in coal mine enterprises," Symmetry, vol. 11, no. 2, Article ID 156, 2019.

[32] Y. Li, Y. Zhang, H. Dai, and Z. Zhao, "Game modelling and strategy research on trilateral evolution for coal-mine operational safety production system: a simulation approach," Complexity, vol. 2020, Article ID 2685238, 2020.

[33] Y. Teng, Y. Wang, J. Wang, and L. Zhang, "Research on decision-making behavior evolution of government, coal mine enterprises and employees under safe education," Eurasia Journal of Mathematics, Science and Technology Education, vol. 13, no. 12, pp. 8267-8281, 2017.

[34] J. Von Neumann and O. Morgenstern, Theory of Games and Economic Behavior, Princeton University Press, Princeton, NJ, USA, 1944.

[35] M. A. Nowak, Evolutionary Dynamics: Exploring the Equations of Life, Harvard University Press, Cambridge, MA, USA, 2006.

[36] M. A. Nowak and K. Sigmund, "Evolutionary dynamics of biological games," Science, vol. 303, no. 5659, pp. 793-799, 2004.

[37] V. R. Venkateswaran and C. S. Gokhale, "Evolutionary dynamics of complex multiple games," Proceedings of the Royal Society B: Biological Sciences, vol. 286, no. 1905, Article ID 20190900, 2019.

[38] K. Fan and E. C. M. Hui, "Evolutionary game theory analysis for understanding the decision-making mechanisms of governments and developers on green building incentives," Building and Environment, vol. 179, Article ID 106972, 2020.

[39] H. Hu, Y. Liu, C. Chen, H. Zhang, and Y. Liu, "Optimal decision making approach for cyber security defense using evolutionary game," IEEE Transactions on Network and Service Management, vol. 17, no. 3, pp. 1683-1700, 2020.

[40] X. Gao, J. Shen, W. He, F. Sun, and Y. Kong, "An evolutionary game analysis of governments' decision-making behaviors and factors influencing watershed ecological compensation in China," Journal of Environmental Management, vol. 251, Article ID 109592, 2019.

[41] W. Shia, H. Wang, C. Chen, and Z. Kong, "Evolutionary game analysis of decision-making dynamics of local governments and residents during wildfires," International Journal of Disaster Risk Reduction, vol. 53, Article ID 101991, 2021.

[42] L. Du, Y. Feng, W. Lu, L. Kong, and Z. Yang, "Evolutionary game analysis of stakeholders' decision-making behaviours in construction and demolition waste management," Environmental Impact Assessment Review, vol. 84, Article ID 106408, 2020.

[43] P. Dirac, The Principles of Quantum Mechanics, Oxford University Press, Oxford, UK, 1930.

[44] J. Von Neumann, Mathematical Foundations of Quantum Mechanics, Princeton University Press, Princeton, NJ, USA, 1955. 
[45] M. Asano, I. Basieva, A. Khrennikov, M. Ohya, and Y. Tanaka, "A quantum-like model of selection behavior," Journal of Mathematical Psychology, vol. 78, pp. 2-12, 2017.

[46] I. Basieva, P. Khrennikova, E. M. Pothos, M. Asano, and A. Khrennikov, "Quantum-like model of subjective expected utility," Journal of Mathematical Economics, vol. 78, pp. 150-162, 2018.

[47] W. Hu and Q. Zhu, "Moment exponential stability of stochastic nonlinear delay systems with impulse effects at random times," International Journal of Robust and Nonlinear Control, vol. 29, no. 12, pp. 3809-3820, 2019.

[48] F. Wu and G. Yin, "An averaging principle for two-time-scale stochastic functional differential equations," Journal of Differential Equations, vol. 269, no. 1, pp. 1037-1077, 2020.

[49] P. D. Taylor and L. B. Jonker, "Evolutionary stable strategies and game dynamics," Mathematical Biosciences, vol. 40, no. 12, pp. $145-156,1978$.

[50] D. Friedman, "On economic applications of evolutionary game theory," Journal of Evolutionary Economics, vol. 8, no. 1, pp. 15-43, 1998. 